\title{
Meldeverfahren im Zusammenhang mit SAE und SUSAR bei klinischen Versuchen mit Arzneimitteln und Medizinprodukten
}

\section{$A G \mathrm{KoBeK}^{*}$}

Die AG KoBeK ist eine informelle Gruppierung mit Vertretern und Vertreterinnen von SAMW, Swissmedic, BAG, der Arbeitsgemeinschaft der Ethikkommissionen AGEK, der Schweizerischen Gesellschaft für biomedizinische Ethik SGBE und der Kantone.
Die Meldung schwerwiegender unerwünschter Ereignisse (serious adverse events; SAE), schwerwiegender unerwünschter Arzneimittelwirkungen (suspected unexpected serious adverse reactions; SUSAR) und schwerwiegender Vorkommnisse mit Medizinprodukten bei klinischen Versuchen wird in der Verordnung über klinische Versuche mit Heilmitteln (VKlin; Art. 22-24) geregelt. Artikel 20 regelt die Sicherheitsmassnahmen, die ergriffen werden müssen, wenn die Sicherheit der Versuchspersonen beeinträchtigt sein könnte. Die Ethikkommissionen werden mit diesen Meldungen überschwemmt. So erhält z.B. die Kantonale Ethikkommission Bern pro Jahr über 7000 Meldungen. Einerseits sind diese Meldungen für die Prüfer und die Sponsoren sehr aufwendig und teuer. Anderseits sind die Ethikkommissionen nicht in der Lage, diese Flut von Meldungen seriös zu bearbeiten. Die AG KoBeK hat in mehreren Sitzungen eine Empfehlung erarbeitet, die die Zahl der Meldungen und die Doppelspurigkeiten im Rahmen der gesetzlichen Vorgaben möglichst reduzieren soll.

\section{Versuche mit Arzneimitteln}

Die Information über SAE oder SUSAR soll nicht in Form kommentarloser Einzelmeldungen erfolgen, sondern in Form einer zusammenfassenden, wertenden Darstellung des vermuteten Arzneimittelrisikos unter Einbezug aller verfügbaren Daten (präklinische, klinische, gegebenenfalls PMS-Daten, relevante Publikationen), insbesondere zu Häufigkeit, Schweregrad, Mechanismus, Risikofaktoren und vorgesehenen risikomindernden Massnahmen. Relevante Einzelmeldungen können beigefügt oder ausnahmsweise auch mit einem entsprechenden Begleitschreiben als Erstinformation über ein neues Risiko verwendet werden.

\section{Versuche mit nur einem Zentrum in der Schweiz}

Monozentrische Versuche und internationale multizentrische Versuche mit nur einem einzigen schweizerischen Zentrum

1.1 SAE (bei Tod einer Versuchsperson; unverzüglich) und SUSAR (bei Tod einer Versuchsperson und Lebensgefahr; innerhalb von 7 Tagen) im eigenen Prüfzentrum werden vom Prüfer direkt der zuständigen Ethikkommission gemeldet (Art. 22, Abs. 3 und Art. 23, Abs. 1 VKlin). Er teilt gegebenenfalls der EK mit, ob Sicherheitsmassnahmen gemäss Art. 20 VKlin erforderlich sind.

1.2 Bei internationalen multizentrischen Studien informiert der Prüfer die EK direkt über SAE und SUSAR (bzw. über sogenannte Sicherheitssignale) aus der Schweiz oder anderen (= ausländischen) Prüfzentren, wenn allenfalls Sicherheitsmassnahmen (inkl. Informationsmassnahmen) gemäss Art. 20 VKlin erforderlich sind.

1.3 Mindestens einmal jährlich legt der Prüfer der EK eine vom Sponsor erstellte Liste mit sämtlichen SAE und SUSAR und einen Bericht über die Sicherheit der Versuchspersonen (Art. 23, Abs. 4 VKlin) vor.

\section{Versuche mit mehreren Zentren in der Schweiz}

2.1 SAE (Tod einer Versuchsperson; unverzüglich) und SUSAR (Tod einer Versuchsperson und Lebensgefahr; innerhalb von 7 Tagen) im eigenen Prüfzentrum werden vom Prüfer direkt der zuständigen Ethikkommission gemeldet. Er teilt gegebenenfalls der EK mit, ob aus seiner Sicht Sicherheitsmassnahmen gemäss Art. 20 VKlin erforderlich sind. 
2.2 Der koordinierende Prüfer informiert die für ihn zuständige EK über alle meldepflichtigen SAE und SUSAR.

2.3 Werden an einem Prüfzentrum Massnahmen nach Art. 12 oder 20 VKlin ergriffen, orientiert der koordinierende Prüfer unverzüglich via die anderen Prüfer alle involvierten EK.

2.4 Der koordinierende Prüfer legt mindestens einmal jährlich jedem Prüfer zuhanden der jeweils zuständigen EK eine vom Sponsor erstellte Liste mit sämtlichen SAE und SUSAR und einen Bericht über die Sicherheit der Versuchspersonen (Art. 23, Abs. 4 VKlin) vor.

\section{Versuche mit Medizinprodukten}

(z.B. Implantat, Gerät, Instrument, medizinisches Verbrauchsmaterial)

1.1 Schwerwiegende Vorkommnisse im eigenen Prüfzentrum, die möglicherweise auf ein Medizinprodukt zurückzuführen sind, werden vom Prüfer innert zehn Tagen der zuständigen EK gemeldet (die Meldepflicht gilt auch für erwartete schwerwiegende Vorkommnisse, bei Tod, schwerwiegende Beeinträchtigungen der Gesundheit, Beinahevorkommnisse mit Gefahren für Versuchspersonen, Anwender, Dritte). Er teilt der EK mit, ob aus seiner Sicht Sicherheitsmassnahmen gemäss Art. 20 VKlin erforderlich sind.

1.2 Werden an einem Prüfzentrum Massnahmen nach Art. 12 oder 20 VKlin ergriffen, orientiert der koordinierende Prüfer unverzüglich via die anderen Prüfer alle involvierten EK.

1.3 Bei internationalen multizentrischen Versuchen informiert der koordinierende Prüfer die EK direkt über solche Vorkommnisse aus anderen (= ausländischen) Prüfzentren, wenn Sicherheitsmassnahmen gemäss Art. 20 VKlin erforderlich sind.

1.4 Mindestens einmal jährlich legt der Prüfer der EK eine Liste mit sämtlichen Vorkommnissen vor. Er erstattet Bericht über die Sicherheit der Versuchspersonen (Art. 24, Abs. 2 VKlin). 